OPEN ACCESS

Edited by:

Mario Ulises Pérez-Zepeda, Dalhousie University, Canada

Reviewed by: Scott Kehler

Dalhousie University, Canada

Marios Kyriazis,

National Gerontology Centre, Cyprus

*Correspondence:

Ji Won Yoon

jwyoonmd@gmail.com orcid.org/0000-0001-9003-0614

tThese authors have contributed equally to this work and share first authorship

Specialty section:

This article was submitted to Geriatric Medicine,

a section of the journal

Frontiers in Medicine

Received: 11 January 2021 Accepted: 05 March 2021 Published: 29 March 2021

Citation:

Chung GE, Park HE, Lee H, Kim MJ,

Choi S-Y, Yim JY and Yoon JW (2021)

Sarcopenic Obesity Is Significantly

Associated With Coronary Artery

Calcification. Front. Med. 8:651961

doi: 10.3389/fmed.2021.651961

\section{Sarcopenic Obesity Is Significantly Associated With Coronary Artery Calcification}

\author{
Goh Eun Chung ${ }^{\dagger}$, Hyo Eun Park ${ }^{\dagger}$, Heesun Lee, Min Joo Kim, Su-Yeon Choi, \\ Jeong Yoon Yim and Ji Won Yoon* \\ Internal Medicine, Seoul National University Hospital Healthcare System Gangnam Center, Seoul, South Korea
}

Background: An association between sarcopenic obesity and cardiovascular disease has been suggested. We investigated the relationship between sarcopenia and coronary atherosclerosis, taking into account the presence or absence of obesity in a health check-up population.

Methods: Data were reviewed for subjects who underwent bioelectrical impedance analysis (BIA) and coronary calcium scoring (CAC) computed tomography between January 2017 and December 2018. Appendicular skeletal muscle mass (ASM) was assessed using BIA. Sarcopenia was defined as reduction of muscle mass and calculated as ASM\% (ASM/body weight) more than two standard deviations below the sex-specific mean for healthy young adults. CAC scores were dichotomized as low $(<100)$ or high ( $\geq 100)$.

Results: Among 1,282 subjects (mean age, 58.1 years; $75.5 \%$ male), the prevalence of high CAC was $21 \%$. When the study population was divided into four groups according to their obesity and sarcopenia status, the prevalence of high CAC in the sarcopenic-obesity (SO) group was significantly higher than in the other groups $(40.7 \%, P<0.001)$. After adjusting for age, sex, hypertension, diabetes, dyslipidemia, and creatinine, subjects with SO exhibited a significantly higher odds of a high CAC score, compared with the non-sarcopenic, non-obese group (odds ratio, 1.92; 95\% confidence interval, 1.16-3.18, $P=0.011$.

Conclusion: SO was significantly associated with CAC, independent of known risk factors for coronary artery disease. These findings suggest that sarcopenia and obesity may potentiate each other to increase atherosclerotic burden in coronary arteries, which may eventually lead to adverse cardiovascular events.

Keywords: sarcopenia, coronary artery calcification, obesity, atherosclerosis, cardiovascular risk

\section{KEY POINTS}

- Sarcopenic obesity is significantly associated with coronary artery calcification, independent of established risk factors for coronary artery disease.

- The combination of obesity and sarcopenia may be associated with an increased risk of coronary atherosclerosis, which may eventually lead to cardiovascular events. 


\section{INTRODUCTION}

Sarcopenia refers to an age-related decline in skeletal muscle mass and strength, with or without a reduction in physical performance (1). The importance of sarcopenia is based on its relationship with metabolic and cardiovascular diseases (2-4), as excess fat mass and reduced lean mass are associated with increased mortality. Thus, sarcopenia and obesity act interactively, resulting in various metabolic and functional impairments.

Sarcopenic obesity (SO) is the state in which sarcopenia and obesity coexist, presenting as both decreased muscle mass and increased adiposity. Previous studies have reported an association between $\mathrm{SO}$ and traditional cardiovascular risk factors, including metabolic syndrome, diabetes, and insulin resistance (5). However, the association between SO and cardiovascular outcomes remains unclear, as studies have shown conflicting results. Stephen and Janssen followed 3,366 community-dwelling elderly individuals with no baseline cardiovascular disease for 8 years. They reported that sarcopenia (defined as reduced muscle strength or mass) and obesity alone did not affect the risk of cardiovascular disease, but SO (defined by reduced muscle strength) was associated with a $23 \%$ increased risk of cardiovascular disease (6). In another study, Farmer et al. investigated the association of sarcopenia (assessed by grip strength) and obesity with cardiovascular disease and showed that obesity alone, sarcopenia alone, and SO were all associated with increased cardiovascular and all-cause mortality, as well as cardiovascular disease events (except sarcopenia alone) (7). These results indeed show that SO is associated with cardiovascular disease.

Coronary artery calcification (CAC) reflects the burden and severity of atherosclerosis in coronary arteries and is a wellknown marker associated with adverse cardiovascular outcomes $(8,9)$. CAC is also used for individual risk stratification and to detect subclinical atherosclerosis. A population study reported a dose-dependent inverse relationship between lower relative muscle mass and CAC score (10). However, the clinical significance of sarcopenia, obesity, and SO in association with CAC remains to be elucidated. In this study, we aimed to investigate the relationship between sarcopenia and CAC, taking into account the presence or absence of obesity, in a health check-up population.

\section{METHODS}

\section{Study Population}

This retrospective observational study included subjects who underwent routine health check-up at the Seoul National University Hospital Healthcare System Gangnam Center between January 2017 and December 2018. The subjects voluntarily attended for a general health check-up, while others were supported by their employer. They were mostly free of symptoms and voluntarily underwent examinations including bioelectrical analysis, CAC-scoring computed tomography (CT) and blood samplings on the same day. Of 1,304 potential study participants, 22 were excluded because of a previous myocardial infarction, past or current angina, or past or current congestive heart failure presumed secondary to coronary artery disease. Therefore, 1,282 subjects were included in the final analysis.

The study protocol followed the guidelines of the Declaration of Helsinki of 1975, as revised in 1983. The protocol was approved by the Institutional Review Board of Seoul National University Hospital (No. 1606-102-771). Informed consent was waived by the board since researchers accessed and analyzed only de-identified data.

\section{Measurement of Clinical and Laboratory Parameters}

Data regarding past medical history, comorbidities, and medications were obtained using subject-recorded questionnaires. Blood pressure was measured twice, and mean values of the two measurements were reported. Hypertension was defined as a blood pressure $\geq 140 / 90 \mathrm{mmHg}$ or receiving antihypertensive medications, and diabetes was defined as a fasting blood glucose $\geq 126 \mathrm{mg} / \mathrm{dL}$ or receiving glucoselowering agents. Subjects taking lipid-lowering agents or with a total cholesterol $\geq 240 \mathrm{mg} / \mathrm{dL}$ were categorized as having hypercholesterolemia (11).

All blood samples were collected after a 12-h overnight fast. Laboratory tests included serum alanine aminotransferase, aspartate aminotransferase, total cholesterol, triglycerides, highdensity lipoprotein cholesterol, fasting glucose, creatinine, and high-sensitivity C-reactive protein. All of these tests were performed using standard laboratory methods.

\section{Anthropometric Measurements}

The methods employed in this study have been previously described in detail (12). Body weight and height were measured using a digital scale, and body mass index (BMI) was calculated by dividing weight $(\mathrm{kg})$ by the squared value of height $\left(\mathrm{m}^{2}\right)$. A well-trained person used a tape to measure the waist circumference at the midpoint between the lower costal margin and anterior superior iliac crest. For assessing body composition, bioelectrical impedance analysis (BIA) was performed using an InBody 720 Body Composition Analyzer (InBody Co., Ltd., Seoul, Korea). During this test, subjects remained in a standing position for 5-10 min with their legs slightly separated and their arms slightly abducted from the trunk. They were instructed to grasp the handles of the analyzer so each extremity contacted the electrodes. Multi-frequency measurements of impedance for each segment (including the trunk and four extremities) were obtained and used to estimate the appendicular skeletal muscle mass (ASM).

\section{Definitions of Sarcopenia and Obesity}

ASM (kg) was calculated as the sum of the lean muscle mass in all four extremities. ASM\% was calculated as ASM/weight $(\mathrm{kg}) * 100$, as modified from Janssen et al. (13). Sarcopenia was defined as reduction of muscle mass and calculated as an ASM\% more than two standard deviations (SDs) below the sexspecific mean for healthy young adults, according to nationwide health examinations of the Korean population (ASM\% $<29.0$ in men and $<22.9$ in women) (14). Obesity was defined as a 
BMI $\geq 25\left(\mathrm{~kg} / \mathrm{m}^{2}\right)$, according to the World Health Organization recommendation for the Asian-Pacific region (15). The subjects were classified into normal (control), obese, sarcopenic, and SO groups according to these definitions.

\section{Measurement of Coronary Artery Calcification}

For all subjects, cardiac CT was performed for screening purposes at the patients' request. CT was conducted using a 256-slice multi-detector CT scanner (Brilliance iCT 256; Philips Medical Systems, Cleveland, OH, USA), with electrocardiogram-gated dose modulation. A standard scanning protocol was used, with $128 \times 0.625 \mathrm{~mm}$ section collimation, $0.27 \mathrm{~ms}$ rotation time, $120 \mathrm{kV}$ tube voltage, and $800 \mathrm{~mA}$ tube current. Data were reconstructed to generate $3-\mathrm{mm}$ thick slices with a $400-\mathrm{ms}$ acquisition window. The CAC score was calculated using a CT software program (Rapidia 2.8; INFINITT, Seoul, Korea) (16).

\section{Statistical Analysis}

The outcome variable was CAC score, which was dichotomized as low (score $<100)$ or high $(\geq 100)$ (16). Continuous variables were expressed as mean $\pm \mathrm{SD}$, and categorical variables were expressed as number and percentage. Comparisons of continuous variables between groups were performed using Student's $t$-test or analysis of variance, and categorical variables were compared using chisquare test or Fisher's exact test. Logistic regression analysis was used to analyze the associations of sarcopenia, obesity, and SO with CAC score, while controlling for potential confounders. Multivariate logistic regression analysis was performed to determine the independent associations of sarcopenia, obesity, and SO with CAC, after adjusting for age, sex, hypertension, diabetes, dyslipidemia, and serum creatinine level. All statistical analyses were performed using SPSS 22.0 (SPSS Inc., Chicago, IL, USA), and $P<0.05$ were considered statistically significant.

\section{RESULTS}

\section{Clinical Characteristics of Study Population}

The mean age of our study population was $58.1 \pm 9.3$ years, and $75.5 \%$ of subjects were male. Among the 1,282 subjects, $21 \%$ had a high CAC score. Clinical characteristics according to CAC score are summarized in Table 1. Compared with the low CAC group, individuals with a high CAC score were older, were more frequently male, and had a higher BMI and higher prevalence of diabetes, hypertension, and dyslipidemia $(P<0.001)$. Total cholesterol and fasting blood glucose levels were also higher in individuals with a high CAC. The prevalence of sarcopenia was significantly higher in the high CAC group than in the low CAC group ( 16.5 vs. $7.4 \%, P<0.05$ ).

Of the study subjects, 122 (9.5\%) had sarcopenia, $522(40.7 \%)$ were obese, and $108(8.4 \%)$ had SO. Table 2 compares the four groups. Subjects with SO were more frequently male, had a higher prevalence hypertension, and exhibited higher serum AST, ALT, and triglyceride levels than those without SO. As shown in Figure 1, the prevalence of high CAC score was significantly
TABLE 1 | Comparison of baseline characteristics according to coronary artery calcification score.

\begin{tabular}{lccc}
\hline & $\begin{array}{c}\text { CACS }<\mathbf{1 0 0} \\
(\mathbf{N}=\mathbf{9 8 5})\end{array}$ & $\begin{array}{c}\text { CACS } \geq \mathbf{1 0 0} \\
(\mathbf{N}=\mathbf{2 9 7})\end{array}$ & $\boldsymbol{P}$-value \\
\hline Age (years) & $56.4 \pm 8.8$ & $62.5 \pm 9.2$ & $<0.001$ \\
Male, $n(\%)$ & $699(71.0)$ & $265(89.2)$ & $<0.001$ \\
BMI (kg/m $\left.{ }^{2}\right)$ & $24.4 \pm 2.9$ & $25.3 \pm 3.2$ & $<0.001$ \\
BMI $\geq 25\left(\mathrm{~kg} / \mathrm{m}^{2}\right)$ & $373(37.9)$ & $149(50.2)$ & $<0.001$ \\
Waist circumference (cm) & $88.8 \pm 8.3$ & $91.7 \pm 8.6$ & $<0.001$ \\
ASM,\% (kg) & $30.7 \pm 3.4$ & $30.9 \pm 3.1$ & 0.418 \\
Sarcopenia, $n(\%)$ & $73(7.4)$ & $49(16.5)$ & $<0.001$ \\
Comorbidities & & & \\
Diabetes mellitus, $n$ (\%) & $68 / 982(6.9)$ & $67(22.6)$ & $<0.001$ \\
Hypertension, $n$ (\%) & $235 / 982(23.9)$ & $160(53.9)$ & $<0.001$ \\
Dyslipidemia, $n$ (\%) & $209 / 983(21.3)$ & $133(44.8)$ & $<0.001$ \\
Laboratory parameters & & & \\
AST (IU/L) & $28.2 \pm 18.0$ & $28.4 \pm 16.0$ & 0.893 \\
ALT (IU/L) & $26.8 \pm 13.1$ & $28.4 \pm 15.9$ & 0.093 \\
Cholesterol (mg/dL) & $195.0 \pm 35.8$ & $181.9 \pm 44.4$ & $<0.001$ \\
Triglyceride (mg/dL) & $125.4 \pm 74.6$ & $120.7 \pm 66.5$ & 0.308 \\
HDL-cholesterol (mg/dL) & $53.1 \pm 13.4$ & $53.3 \pm 14.6$ & 0.816 \\
Fasting glucose (mg/dL) & $103.9 \pm 17.7$ & $113.0 \pm 26.6$ & $<0.001$ \\
Creatinine (mg/dL) & $0.87 \pm 0.2$ & $0.91 \pm 0.2$ & 0.001 \\
HS-CRP (mg/dL) & $0.16 \pm 0.6$ & $0.15 \pm 0.4$ & 0.757 \\
& & &
\end{tabular}

Data are shown as the mean $\pm S D$.

CACS, coronary artery calcification score; BMI, body mass index; AST, aspartate aminotransferase; ALT, alanine aminotransferase; ASM, appendicular muscle mass; HDL, high-density lipoprotein; HS-CRP, high sensitivity C-reactive protein.

higher in subjects with $\mathrm{SO}$, compared with the other groups $(P<0.001$ for trend).

\section{Sarcopenia With or Without Obesity and Coronary Artery Calcium Score}

An analysis of the relationship between sarcopenia (with or without obesity) and CAC score is shown in Table 3. Three different multivariate models were constructed, adjusting for traditional atherosclerosis risk factors. When adjusting for age and sex (Model I), obesity and SO were associated with an increased risk of coronary calcification [odds ratio (OR), 1.54; 95\% confidence interval (CI), 1.13-2.11, and OR, 2.61; 95\% CI, 1.64-4.16, respectively]. As shown in Models II and III, additional adjustments with other traditional cardiovascular risk factors attenuated the correlation between SO and CAC, but in all three models, SO remained a statistically and clinically significant parameter associated with increased odds of a high CAC score (Model II: OR, 1.92; 95\% CI, 1.16-3.18; $P=0.009$; Model III: OR, $1.92 ; 95 \% \mathrm{CI}, 1.16-3.18 ; P=0.011)$. However, the association with obesity alone no longer remained statistically significant after additional covariate adjustments (Model II: $P=0.058$; Model III: $P=0.114$; Table 3). When we performed stratified analysis according to sex and age groups, the association between $\mathrm{SO}$ and CAC was significant only in men (Table 4). 
TABLE 2 | Comparison of baseline characteristics according to sarcopenia with/without obesity.

\begin{tabular}{|c|c|c|c|c|c|}
\hline & $\begin{array}{c}\text { Control } \\
(N=746)\end{array}$ & $\begin{array}{l}\text { Obesity } \\
(N=414)\end{array}$ & $\begin{array}{l}\text { Sarcopenia } \\
(N=14)\end{array}$ & $\begin{array}{c}\text { SO } \\
(N=108)\end{array}$ & $P$-value* \\
\hline Age (years) & $58.6 \pm 8.8$ & $56.5 \pm 9.9$ & $64.5 \pm 8.1$ & $59.2 \pm 10.2$ & $<0.001$ \\
\hline Diabetes mellitus, $n(\%)$ & 69/745 (9.3) & $39 / 413(9.4)$ & $4(28.6)$ & 23/107 (21.5) & $<0.001$ \\
\hline Hypertension, $n$ (\%) & 174/746 (23.3) & $154 / 412(37.4)$ & $4(28.6)$ & 63/107 (58.9) & $<0.001$ \\
\hline ALT (IU/L) & $26.2 \pm 12.6$ & $27.8 \pm 13.2$ & $26.7 \pm 7.7$ & $30.1 \pm 14.7$ & 0.013 \\
\hline Cholesterol (mg/dL) & $191.8 \pm 38.0$ & $188.3 \pm 38.6$ & $175.3 \pm 43.1$ & $197.0 \pm 44.6$ & 0.055 \\
\hline Triglyceride (mg/dL) & $106.2 \pm 57.5$ & $147.1 \pm 79.4$ & $135.5 \pm 69.2$ & $151.3 \pm 96.7$ & $<0.001$ \\
\hline HDL cholesterol (mg/dL) & $56.0 \pm 13.9$ & $49.4 \pm 12.5$ & $52.7 \pm 21.7$ & $49.7 \pm 11.9$ & $<0.001$ \\
\hline Fasting glucose (mg/dL) & $103.6 \pm 17.7$ & $107.6 \pm 20.0$ & $125.1 \pm 38.0$ & $116.7 \pm 30.8$ & $<0.001$ \\
\hline
\end{tabular}

Data are shown as the mean $\pm S D$.

SO, sarcopenia-obesity; HDL, high-density lipoprotein; HS-CRP, high sensitivity C-reactive protein; CACS, coronary artery calcification score.

${ }^{*} P$-value for test of trend of odds.

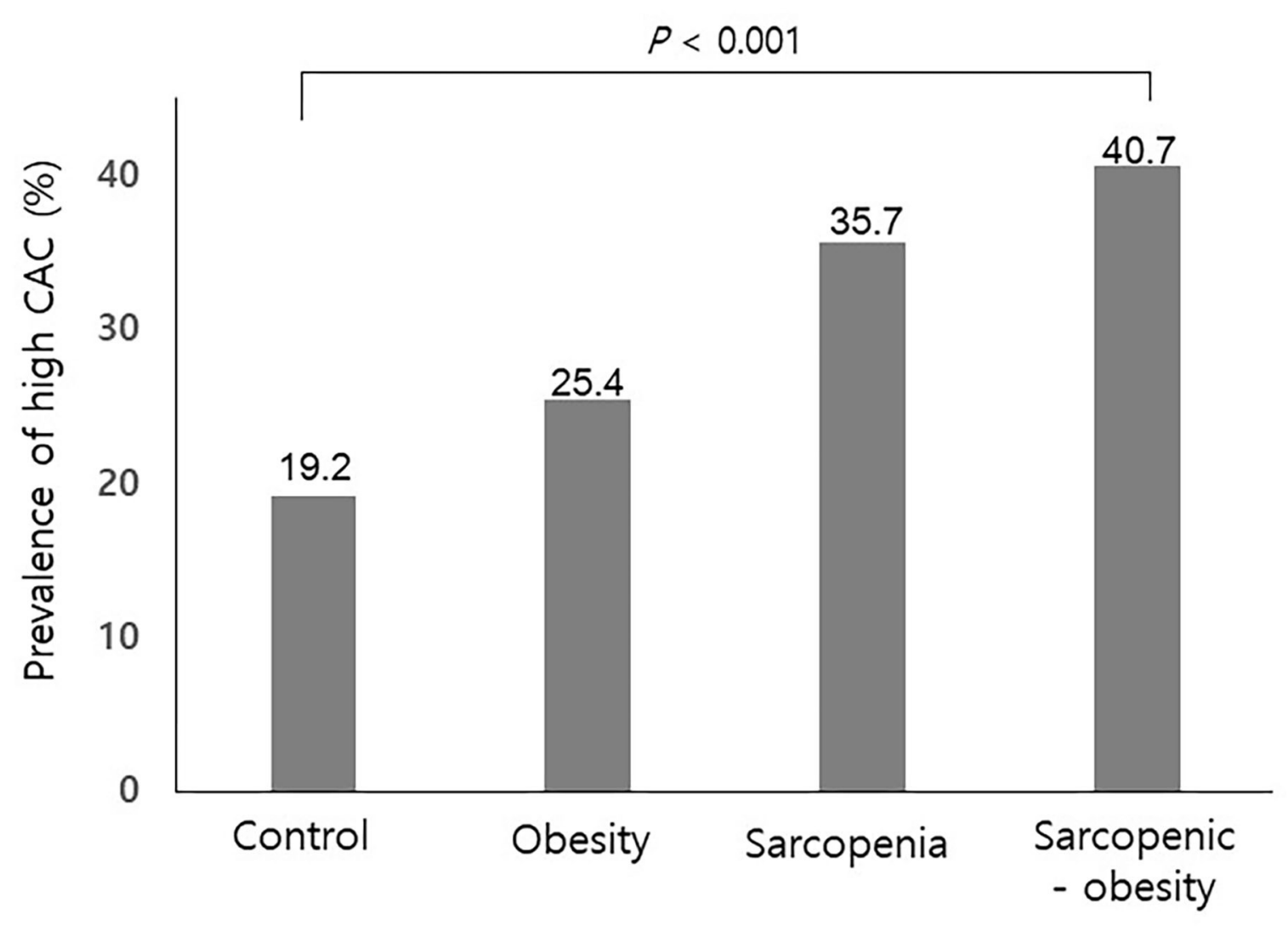

FIGURE 1 | Prevalence of a high coronary artery calcification score according to sarcopenia with or without obesity. CAC, coronary artery calcification. $P$ for trend.

We then evaluated the role of obesity in the association between CAC and sarcopenia. The prevalence of obesity was $40.7 \%$ in our study population. In multivariate analysis, obesity alone was significantly associated with an increased risk of CAC (Supplementary Table 1). Thus, we divided obese subjects into obesity without sarcopenia and obesity with sarcopenia (i.e., 
TABLE 3 | Multivariate analyses of the risk for coronary artery calcification according to sarcopenia with or without obesity.

\begin{tabular}{|c|c|c|c|c|c|c|}
\hline & $\begin{array}{l}\text { Model I } \\
\text { OR }(95 \% \mathrm{Cl})\end{array}$ & $P$-value & $\begin{array}{l}\text { Model II } \\
\text { OR (95\% Cl) }\end{array}$ & $P$-value & $\begin{array}{l}\text { Model III } \\
\text { OR }(95 \% \mathrm{Cl})\end{array}$ & $P$-value \\
\hline Control & 1 (ref) & $<0.001^{*}$ & 1 (ref) & $0.041^{*}$ & 1 (ref) & $0.064^{*}$ \\
\hline Sarcopenia & $1.58(0.47 \sim 5.29)$ & 0.455 & $1.40(0.36 \sim 5.43)$ & 0.627 & $1.33(0.36 \sim 4.97)$ & 0.670 \\
\hline Sarcopenia with obesity & $2.61(1.64 \sim 4.16)$ & $<0.001$ & $1.92(1.18-3.13)$ & 0.009 & $1.92(1.16-3.18)$ & 0.011 \\
\hline
\end{tabular}

OR, odds ratio; $\mathrm{Cl}$, confidence interval.

Model I: adjusted for age and sex.

Model II: adjusted for age, sex, hypertension, and diabetes.

Model III: adjusted for age, sex, hypertension, diabetes, dyslipidemia, and creatinine.

${ }^{*} P$-value for test of trend of odds.

TABLE 4 | Subgroup analysis of the risk for coronary artery calcification according to sarcopenia with or without obesity.

\begin{tabular}{|c|c|c|c|c|}
\hline & \multicolumn{2}{|l|}{ Men } & \multicolumn{2}{|l|}{ Women } \\
\hline & OR (95\% Cl) & $P$-value & OR (95\% Cl) & $P$-value \\
\hline Control & 1 (ref) & $0.035^{\star}$ & 1 (ref) & $0.443^{\star}$ \\
\hline Obesity & $1.31(0.92-1.88)$ & 0.138 & $1.49(0.59-3.76)$ & 0.400 \\
\hline Sarcopenia & $0.99(0.23-4.36)$ & 0.993 & $3.57(0.28-45.31)$ & 0.326 \\
\hline \multirow[t]{2}{*}{ Sarcopenia with obesity } & $2.20(1.28-3.78)$ & $<0.001$ & $0.35(0.04-3.08)$ & 0.342 \\
\hline & \multicolumn{2}{|l|}{ Age $<65$} & \multicolumn{2}{|l|}{ Age $\geq 65$} \\
\hline Control & 1 (ref) & $0.463^{\star}$ & 1 (ref) & $0.479^{*}$ \\
\hline Obesity & $1.22(0.83-1.80)$ & 0.312 & $1.06(0.56-2.03)$ & 0.855 \\
\hline Sarcopenia & $1.18(0.14-10.31)$ & 0.879 & $1.87(0.29-12.00)$ & 0.509 \\
\hline Sarcopenia with obesity & $1.58(0.10-0.43)$ & 0.134 & $1.96(0.80-4.78)$ & 0.142 \\
\hline
\end{tabular}

OR, odds ratio; $\mathrm{Cl}$, confidence interval.

Adjusted for age, sex, hypertension, diabetes, dyslipidemia, and creatinine.

${ }^{*} P$-value for test of trend of odds.

SO). In the age- and sex-adjusted Model I, obesity without sarcopenia and SO were both associated with an increased odds of a high CAC score (OR, 1.53; 95\% CI, 1.12-2.09 and OR, 2.58; 95\% CI, 1.62-4.11, respectively). However, when adjusted for additional cardiovascular risk factors, only $\mathrm{SO}$ associations remained statistically significant (Model II: OR, 1.91; 95\% CI, 1.17-3.13; $P=0.010$ and Model III: OR, 1.91; 95\% CI, 1.15-3.16; $P=0.012$; Table 5).

\section{DISCUSSION}

As shown in the present study, patients with SO have a higher risk of CAC. A significant association was found between SO and a high CAC score, independent of established risk factors for coronary artery disease. These findings suggest that the combination of obesity and sarcopenia may be associated with an increased risk of developing coronary atherosclerosis.

Reported prevalence of sarcopenia has varied according to the definitions used and populations studied. The prevalence has ranged from 5 to $13 \%$ in patients above 60 years of age $(17,18)$.
Results of previous studies of Korean populations have reported sarcopenia prevalence of $5.1 \%$ in men and $14.2 \%$ in women aged $\geq 60$ years (19), and $9.7 \%$ in men and $11.8 \%$ in women aged $\geq 65$ years (14). These values are generally similar to the results of the current study, in which we found a $9.7 \%$ overall prevalence of sarcopenia, using the definition modified from Janssen et al. (13), in our Korean subjects with no known coronary artery disease and a mean age of 58 years. The prevalence of SO was $8.4 \%$ in our study population, which was similar to the prevalence reported in one previous study $(5.1 \%$ in men and $12.5 \%$ in women aged $\geq 60$ years) (19) and lower than the values reported in another study (18.3 and $26.6 \%$ in women) (20). Differences in prevalence likely reflect varying definitions of $\mathrm{SO}$ and different study populations.

Obesity is a well-established major risk factor for cardiovascular disease (21-23). However, obesity defined by BMI does not account for wide variations in body fat distribution and may not correspond to the same degree of fatness or associated health risks in different individuals and populations. A previous study reported that sarcopenia but not excess weight or total caloric intake was associated with subclinical atherosclerosis in elderly patients (24). Thus, considering sarcopenia in addition to obesity may increase the accuracy of predicting cardiovascular disease risk.

Various studies have investigated the clinical significance of sarcopenia and $\mathrm{SO}$ in relation to cardiovascular disease (5). In a Japanese population study, sarcopenia was associated with greater arterial stiffness in women, suggesting that sarcopenia is associated with risk factors for atherosclerosis (25). Health interview survey population data likewise showed that sarcopenia was independently associated with cardiovascular events (3). Moreover, SO was associated with a higher risk for dyslipidemia (20), metabolic syndrome (26), diabetes (27), and hypertension (28) than sarcopenia or obesity alone. In our study, non-sarcopenic obese and non-obese sarcopenic groups did not have a significantly increased odds of a high CAC score in multivariate analysis. This suggests that obesity and sarcopenia alone may be insufficient to significantly increase the risk of developing atherosclerosis. Although not statistically significant, the non-sarcopenic obese and nonobese sarcopenic groups showed similar increases in OR for a high CAC score, and the SO group exhibited an even 
TABLE 5 | Multivariate analyses of the risk for coronary artery calcification according to obesity with or without sarcopenia.

\begin{tabular}{|c|c|c|c|c|c|c|}
\hline & $\begin{array}{c}\text { Model I } \\
\text { OR (95\% Cl) }\end{array}$ & $P$-value & $\begin{array}{c}\text { Model II } \\
\text { OR (95\% CI) }\end{array}$ & $P$-value & $\begin{array}{c}\text { Model III } \\
\text { OR (95\% CI) }\end{array}$ & $P$-value \\
\hline Control & 1 (ref) & $<0.001^{*}$ & 1 (ref) & $0.018^{\star}$ & 1 (ref) & $0.029^{\star}$ \\
\hline Obesity without sarcopenia & 1.53 (1.12-2.09) & 0.008 & $1.36(0.98-1.88)$ & 0.062 & $1.30(0.93-1.81)$ & 0.121 \\
\hline Obesity with sarcopenia & $2.58(1.62 \sim 4.11)$ & $<0.001$ & $1.91(1.17-3.13)$ & 0.010 & $1.91(1.15-3.16)$ & 0.012 \\
\hline
\end{tabular}

CAC, coronary artery calcification score; OR, odds ratio; Cl, confidence interval.

Model I: adjusted for age and sex.

Model II: adjusted for age, sex, hypertension, and diabetes.

Model III: adjusted for age, sex, hypertension, diabetes, dyslipidemia, and creatinine.

${ }^{\star} P$-value for test of trend of odds.

higher OR, suggesting that obesity and sarcopenia may exert additive or even synergistic effects on the development of CAC. In this study, the association between SO and CAC was significant only in men, suggesting the different effect depending on gender.

CT-based screening evaluation has become an important role in detecting subclinical atherosclerosis, which has shown incremental predictive value over known risk factors. CAC is a useful tool for individualized-risk stratification and outcome prediction (29-31). The novel finding of the current study is the independent association between SO and CAC, which was not seen with sarcopenia alone or obesity alone. SO itself was significantly associated with CAC, reflecting greater risk and burden of atherosclerotic changes in people with SO.

The underlying mechanism of the close link between SO and coronary atherosclerosis is not fully understood. Loss of skeletal muscle reduces the mass of the primary tissue responsible for insulin-mediated glucose disposal $(17,32)$ and promotes insulin resistance, which plays a key role in the pathogenesis of atherosclerosis. Increased muscle strength has been associated with reduced blood pressure and improved hemodynamics (33), suggesting that muscle exerts a protective role in the development of atherosclerosis. In addition, obesity promotes inflammation by increasing pro-inflammatory cytokines, such as tumor necrosis factor-alpha and interleukin-6, and increases cardiovascular disease risk $(34,35)$. These inflammatory cytokines induce muscle atrophy and are found quite consistently in sarcopenia $(34,35)$. In a previous study, cellular interleukin6 production and serum insulin-like growth factor-1 levels were significant predictors of sarcopenia, suggesting that they play an important role as inflammatory cytokines (36). Thus, obesity may aggravate sarcopenia, and sarcopenia may exacerbate obesity by decreased metabolic rate and myokine deficiency (37); this vicious cycle may exert synergistic detrimental effects on cardiovascular risk.

\section{LIMITATIONS}

This study has some limitations. First, its cross-sectional design limits the ability to verify causality. Thus, we could not infer causal relationships from this study. Second, BIA is not the gold standard method to evaluate muscle mass. However, it is a very useful and practical tool for screening purposes to evaluate muscle and fat mass, especially in routine clinical evaluations. Third, although sarcopenia is not simply a measure of skeletal mass but also of strength which is a component of the new diagnostic criteria for sarcopenia (38), we could not evaluate muscle strength (e.g., grip strength) or physical performance in this study. Lastly, our study population, who underwent health evaluations upon their own initiative, may not represent the general Korean population. Our results, therefore, should be interpreted with caution.

\section{CONCLUSION}

Subjects with SO have a higher risk for coronary atherosclerosis, as exemplified by higher CAC scores. Obesity alone and sarcopenia alone were not associated with high CAC scores. These results suggest that sarcopenia and obesity may potentiate each other to increase atherosclerotic burden in coronary arteries, which may eventually lead to adverse cardiovascular events. Further studies are warranted to understand the mechanism of association between SO and atherosclerosis and verify a causal relationship between $\mathrm{SO}$ and coronary artery calcification and disease.

\section{DATA AVAILABILITY STATEMENT}

The raw data supporting the conclusions of this article will be made available by the authors, without undue reservation.

\section{ETHICS STATEMENT}

The studies involving human participants were reviewed and approved by Institutional Review Board of Seoul National University Hospital. Written informed consent for participation was not required for this study in accordance with the national legislation and the institutional requirements. 


\section{AUTHOR CONTRIBUTIONS}

GC, HP and JWY conceived the idea, determined the study design, collected the data, and drafted and revised the manuscript. $\mathrm{HL}$ and $\mathrm{MK}$ collected the data, performed the statistical analysis, and revised the manuscript. S-YC and JYY collected and reviewed the data and revised the manuscript. All authors contributed to the article and approved the submitted version.

\section{REFERENCES}

1. Chen LK, Liu LK, Woo J, Assantachai P, Auyeung TW, Bahyah KS, et al. Sarcopenia in Asia: consensus report of the Asian working group for sarcopenia. J Am Med Dir Assoc. (2014) 15:95-101. doi: 10.1016/j.jamda.2013.11.025

2. Rosenberg IH. Sarcopenia: origins and clinical relevance. Clin Geriatr Med. (2011) 27:337-9. doi: 10.1016/j.cger.2011.03.003

3. Chin SO, Rhee SY, Chon S, Hwang YC, Jeong IK, Oh S, et al. Sarcopenia is independently associated with cardiovascular disease in older Korean adults: the Korea national health and nutrition examination survey (KNHANES) from 2009. PLoS ONE. (2013) 8:e60119. doi: 10.1371/journal.pone. 0060119

4. Kim TN, Park MS, Yang SJ, Yoo HJ, Kang HJ, Song W, et al. Prevalence and determinant factors of sarcopenia in patients with type 2 diabetes: the Korean sarcopenic obesity Study (KSOS). Diabetes Care. (2010) 33:14971499. doi: $10.2337 / \mathrm{dc} 09-2310$

5. Atkins JL, Wannamathee SG. Sarcopenic obesity in ageing: cardiovascular outcomes and mortality. Br J Nutr. (2020) 124:1102-13. doi: 10.1017/S0007114520002172

6. Stephen WC, Janssen I. Sarcopenic-obesity and cardiovascular disease risk in the elderly. J Nutr Health Aging. (2009) 13:4606. doi: 10.1007/s12603-009-0084-z

7. Farmer RE, Mathur R, Schmidt AF, Bhaskaran K, Fatemifar G, Eastwood $\mathrm{SV}$, et al. Associations between measures of sarcopenic obesity and risk of cardiovascular disease and mortality: a cohort study and mendelian randomization analysis using the UK biobank. J Am Heart Assoc. (2019) 8:e011638. doi: 10.1161/JAHA.118.011638

8. Otton JM, Yu CY, McCrohon J, Sammel N, Feneley M. Accuracy and clinical outcomes of computed tomography coronary angiography in the presence of a high coronary calcium score. Heart Lung Circ. (2013) 22:9806. doi: 10.1016/j.hlc.2013.05.647

9. Hou ZH, Lu B, Gao Y, Jiang SL, Wang Y, Li W, et al. Prognostic value of coronary CT angiography and calcium score for major adverse cardiac events in outpatients. JACC Cardiovasc Imaging. (2012) 5:9909. doi: 10.1016/j.jcmg.2012.06.006

10. Ko BJ, Chang Y, Jung HS, Yun KE, Kim CW, Park HS, et al. Relationship between low relative muscle mass and coronary artery calcification in healthy adults. Arterioscler Thromb Vasc Biol. (2016) 36:101621. doi: 10.1161/ATVBAHA.116.307156

11. Park HE, Lee H, Choi SY, Kwak MS, Yang JI, Yim JY, et al. Clinical significance of hepatic steatosis according to coronary plaque morphology: assessment using controlled attenuation parameter. J Gastroenterol. (2019) 54:271-80. doi: 10.1007/s00535-018-1516-5

12. Chung GE, Kim MJ, Yim JY, Kim JS, Yoon JW. Sarcopenia is significantly associated with presence and severity of nonalcoholic fatty liver disease. J Obes Metab Syndr. (2019) 28:129-38. doi: 10.7570/jomes.2019.28.2.129

13. Janssen I, Heymsfield SB, Ross R. Low relative skeletal muscle mass (sarcopenia) in older persons is associated with functional impairment and physical disability. J Am Geriatr Sock. (2002) 50:889-96. doi: 10.1046/j.1532-5415.2002.50216.x

14. Kim YS, Lee Y, Chung YS, Lee DJ, Joo NS, Hong D, et al. Prevalence of sarcopenia and sarcopenic obesity in the Korean population based on the
ACKNOWLEDGMENTS

The authors are grateful to $\mathrm{Gu}$ Cheol Jung for help with the statistical analysis.

\section{SUPPLEMENTARY MATERIAL}

The Supplementary Material for this article can be found online at: https://www.frontiersin.org/articles/10.3389/fmed. 2021.651961/full\#supplementary-material

fourth Korean national health and nutritional examination surveys. J Gerontol A Biol Sci Med Sci. (2012) 67:1107-13. doi: 10.1093/gerona/gls071

15. Kim MK, Lee WY, Kang JH, Kang JH, Kim BT, Kim SM, et al. 2014 clinical practice guidelines for overweight and obesity in Korea. Endocrinol Metab. (2014) 29:405-9. doi: 10.3803/EnM.2014.29.4.405

16. Kim D, Choi SY, Park EH, Lee W, Kang JH, Kim W, et al. Nonalcoholic fatty liver disease is associated with coronary artery calcification. Hepatology. (2012) 56:605-13. doi: 10.1002/hep.25593

17. Wang C, Bai L. Sarcopenia in the elderly: basic and clinical issues. Geriatr Gerontol Int. (2012) 12:388-96. doi: 10.1111/j.1447-0594.2012.00851.x

18. Morley JE. Sarcopenia: diagnosis and treatment. J Nutr Health Aging. (2008) 12:452-6. doi: 10.1007/BF02982705

19. Kim TN, Yang SJ, Yoo HJ, Lim KI, Kang HJ, Song W, et al. Prevalence of sarcopenia and sarcopenic obesity in Korean adults: the Korean sarcopenic obesity study. Int J Obes. (2009) 33:885-92. doi: 10.1038/ijo.2009.130

20. Baek SJ, Nam GE, Han KD, Choi SW, Jung SW, Bok AR, et al. Sarcopenia and sarcopenic obesity and their association with dyslipidemia in Korean elderly men: the 2008-2010 Korea national health and nutrition examination survey. J Endocrinol Invest. (2014) 37:247-60. doi: 10.1007/s40618-013-0011-3

21. Khan SS, Ning H, Wilkins JT, Allen N, Carnethon M, Berry JD, et al. Association of body mass index with lifetime risk of cardiovascular disease and compression of morbidity. JAMA Cardiol. (2018) 3:2807. doi: 10.1001/jamacardio.2018.0022

22. Wilson PW, D’Agostino RB, Sullivan L, Parise H, Kannel WB. Overweight and obesity as determinants of cardiovascular risk: the Framingham experience. Arch Intern Med. (2002) 162:1867-72. doi: 10.1001/archinte.162.16.1867

23. Abdullah A, Wolfe R, Stoelwinder JU, de Courten M, Stevenson C, Walls HL, et al. The number of years lived with obesity and the risk of all-cause and cause-specific mortality. Int J Epidemiol. (2011) 40:98596. doi: 10.1093/ije/dyr018

24. Campos AM, Moura FA, Santos SN, Freitas WM, Sposito AC, Brasilia Study on Healthy Aging and Brasilia Heart Study. Sarcopenia, but not excess weight or increased caloric intake, is associated with coronary subclinical atherosclerosis in the very elderly. Atherosclerosis. (2017) 258:13844. doi: 10.1016/j.atherosclerosis.2017.01.005

25. Sanada K, Miyachi M, Tanimoto M, Yamamoto K, Murakami H, Okumura $\mathrm{S}$, et al. A cross-sectional study of sarcopenia in Japanese men and women: reference values and association with cardiovascular risk factors. Eur J Appl Physiol. (2010) 110:57-65. doi: 10.1007/s00421-010-1473-Z

26. Lim S, Kim JH, Yoon JW, Kang SM, Choi SH, Park YJ, et al. Sarcopenic obesity: prevalence and association with metabolic syndrome in the Korean longitudinal study on health and aging (KLoSHA). Diabetes Care. (2010) 33:1652-4. doi: 10.2337/dc10-0107

27. Khadra D, Itani L, Tannir H, Kreidieh D, El Masri D, Ghoch ME. Association between sarcopenic obesity and higher risk of type 2 diabetes in adults: A systematic review and meta-analysis. World J Diabetes. (2019) 10:31123. doi: 10.4239/wjd.v10.i5.311

28. Park SH, Park JH, Song PS, Kim DK, Kim KH, Seol SH, et al. Sarcopenic obesity as an independent risk factor of hypertension. J Am Soc Hypertens. (2013) 7:420-5. doi: 10.1016/j.jash.2013.06.002

29. Greenland P, Bonow RO, Brundage BH, Budoff MJ, Eisenberg MJ, Grundy SM, et al. ACCF/AHA 2007 clinical expert consensus document on coronary artery calcium scoring by computed tomography in global cardiovascular 
risk assessment and in evaluation of patients with chest pain: a report of the American college of cardiology foundation clinical expert consensus task force (ACCF/AHA writing committee to update the 2000 Expert consensus document on electron beam computed tomography) developed in collaboration with the society of atherosclerosis imaging and prevention and the society of cardiovascular computed tomography. J Am Coll Cardiol. (2007) 49:378-402. doi: 10.1016/j.jacc.2006.10.001

30. Bonow RO. Clinical practice. Should coronary calcium screening be used in cardiovascular prevention strategies? N Engl J Med. (2009) 361:9907. doi: 10.1056/NEJMcp0902177

31. Greenland P, LaBree L, Azen SP, Doherty TM, Detrano RC. Coronary artery calcium score combined with Framingham score for risk prediction in asymptomatic individuals. JAMA. (2004) 291:210-5. doi: 10.1001/jama.291.2.210

32. Abbatecola AM, Paolisso G, Fattoretti P, Evans WJ, Fiore V, Dicioccio L, et al. Discovering pathways of sarcopenia in older adults: a role for insulin resistance on mitochondria dysfunction. J Nutr Health Aging. (2011) 15:8905. doi: 10.1007/s12603-011-0366-0

33. Wilmore JH, Stanforth PR, Gagnon J, Rice T, Mandel S, Leon AS, et al. Heart rate and blood pressure changes with endurance training: the HERITAGE family study. Med Sci Sports Exerc. (2001) 33:10716. doi: 10.1097/00005768-200101000-00017

34. Beyer I, Mets T, Bautmans I. Chronic low-grade inflammation and agerelated sarcopenia. Curr Opin Clin Nutr Metab Care. (2012) 15:1222. doi: 10.1097/MCO.0b013e32834dd297
35. Schrager MA, Metter EJ, Simonsick E, Ble A, Bandinelli S, Lauretani F, et al. Sarcopenic obesity and inflammation in the InCHIANTI study. J Appl. Physiol. (2007) 102:919-25. doi: 10.1152/japplphysiol.00627.2006

36. Payette H, Roubenoff R, Jacques PF, Dinarello CA, Wilson PW, Abad LW, et al. Insulin-like growth factor- 1 and interleukin 6 predict sarcopenia in very old community-living men and women: the Framingham heart study. J Am Geriatr Soc. (2003) 51:1237-43. doi: 10.1046/j.1532-5415.2003.51407.x

37. Hong SH, Choi KM. Sarcopenic obesity, insulin resistance, and their implications in cardiovascular and metabolic consequences. Int J Mol Sci. (2020) 21:494. doi: 10.3390/ijms21020494

38. Cruz-Jentoft AJ, Bahat G, Bauer J, Boirie Y, Bruyere O, Cederholm T, et al. Sarcopenia: revised European consensus on definition and diagnosis. Age Ageing. (2019) 48:16-31. doi: 10.1093/ageing/afy169

Conflict of Interest: The authors declare that the research was conducted in the absence of any commercial or financial relationships that could be construed as a potential conflict of interest.

Copyright (c) 2021 Chung, Park, Lee, Kim, Choi, Yim and Yoon. This is an open-access article distributed under the terms of the Creative Commons Attribution License (CC $B Y)$. The use, distribution or reproduction in other forums is permitted, provided the original author(s) and the copyright owner(s) are credited and that the original publication in this journal is cited, in accordance with accepted academic practice. No use, distribution or reproduction is permitted which does not comply with these terms. 